\title{
Modulating Protein Alignment in a Liquid-Crystalline Medium through Conservative Mutagenesis
}

\author{
Lishan Yao and Ad Bax* \\ Laboratory of Chemical Physics, National Institute of Diabetes and Digestive and Kidney Deseases, National \\ Institutes of Health, Bethesda, Maryland 20892-0520 \\ Received May 31, 2007; E-mail: bax@nih.gov
}

Residual dipolar couplings (RDCs) readily can be measured for molecules aligned in a magnetic field and carry a wealth of information on both molecular structure and internal dynamics. ${ }^{1,2}$ Introduction of suitable alignment media, mostly lyotropic liquid crystals, but also anisotropically compressed hydrogels, ${ }^{3,4}$ have enabled accurate NMR measurement of RDCs in isotopically enriched proteins, where they are becoming increasingly used to evaluate and/or refine structural quality and to probe internal dynamics. ${ }^{5-11}$ RDCs report on the time-averaged orientations of the corresponding internuclear vectors, but infinite sets of solutions exist if measurements are carried out in only a single alignment medium. These degeneracies can be lifted if measurements are repeated under conditions where the average alignment direction of the protein relative to the magnetic field has been altered. ${ }^{12,13}$ Up to five linearly independent alignment tensors can be generated, each defined by the five unique elements of their traceless, symmetric $3 \times 3$ Saupe matrix, and if more than five alignments are available, this permits structure-independent validation of the self-consistency of these data. ${ }^{14}$

Availability of five independent alignments permits not only extraction of the motional amplitude of each internuclear vector defined by RDCs, but also reports on the degree of asymmetry and the direction of such motion. ${ }^{5-11}$ Motions probed by RDCs are integrated over a wide range of time scales, from femto- to milliseconds, and RDCs thereby provide a powerful complement to NMR relaxation rates. In recent years, much effort has focused on generating the requisite 5 independent alignment tensors, in some cases using as many as 30 different types of alignment media. ${ }^{15}$ However, with protein alignment being dominated by essentially three independent types of interactions, steric repulsion, electrostatic repulsion, and electrostatic attraction, the generation of five orthogonal alignments has remained quite challenging. ${ }^{16}$

In charged nematic media, such as Pf1 phage, ${ }^{17}$ the alignment tensor is dominated by an electrostatic interaction between the solute and the nematogen. This allows the alignment of a protein in the electric field of the phage to be predicted on the basis of the protein's three-dimensional structure and known location of its charged surface groups. ${ }^{18}$ The present study suggests a rational way to alter the alignment of a protein by changing the protein's surface charge distribution. We demonstrate that conservative mutation of selected surface side chains is an effective method for generating linearly independent alignment tensors. Our study focuses on the third immunoglobulin binding domain of protein G, or GB3, a domain of 56 residues that has been extensively characterized by high-resolution X-ray crystallography ${ }^{19}$ and NMR. ${ }^{5,9,20}$ Its X-ray structure indicates that, despite six mutations, its backbone structure is virtually indistinguishable from GB1, immediately identifying loci for mutation to alter the surface charge distribution without affecting the protein backbone.

11326 - J. AM. CHEM. SOC. 2007, 129, 11326-11327

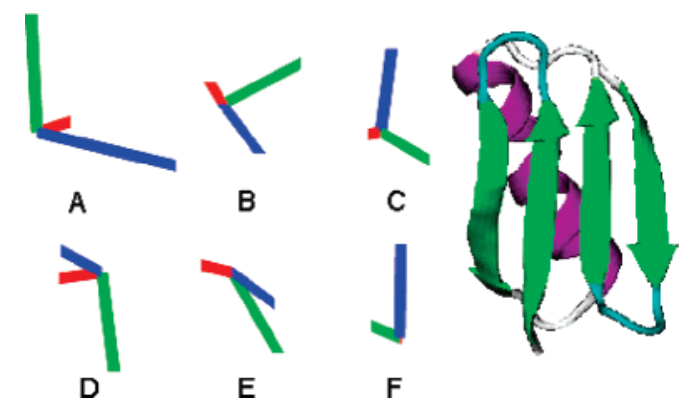

Figure 1. Alignment tensor orientations relative to the ribbon backbone structure of GB3 for six mutants, all in liquid-crystalline Pf1 medium. The six tensors are for (A) K19AD47K; (B) K19ED40N; (C) K19EK4A-CHis-tag; (D) K19EK4A-N-His-tag; (E) K19AT11K; (F) K19EK4A. Diagonalized tensor elements, $\mathrm{D}_{x x}$ (red), $\mathrm{D}_{y y}$ (green) and $\mathrm{D}_{z z}$ (blue) have magnitudes proportional to the length of the corresponding lines.

Our study uses highly robust and commercially available liquidcrystalline suspensions of Pf1 for protein alignment, resulting in NMR spectra of superb quality. Before designing new GB3 mutants, the V42E mutation, also occurring in GB1, was used to increase the net negative charge from -2 to -3 . This mutation increases the alignment contribution from electrostatic repulsion between Pf1 and GB3 and facilitates design of suitable mutations. Using the PALES program, ${ }^{18}$ six mutants that perturb the surface charge distribution were generated: K19AD47K, K19ED40N, K19AT11K, K19EK4A, and K19EK4A with either an N-terminal or a C-terminal His-tag extension. This set yields five independent alignment tensors while minimizing structural perturbation caused by the mutations (Supporting Information); the sixth mutant permits evaluation to what extent five tensors suffice to describe the data and also resolves several cases where very minor chemical shift changes resulted in accidental spectral overlap for some of the mutants. ${ }^{11,14}$

Backbone amide ${ }^{15} \mathrm{~N}-\mathrm{H}$ RDCs were collected by recording $3 \mathrm{D}$ $\mathrm{HNCO}$ spectra without ${ }^{1} \mathrm{H}$ decoupling during ${ }^{15} \mathrm{~N}$ evolution and without ${ }^{13} \mathrm{C}^{\alpha}$ decoupling during $\mathrm{C}^{\prime}$ evolution. In the $3 \mathrm{D} \mathrm{HNCO}$ spectra, each correlation is split into four multiplet components, separated by ${ }^{1} J_{\mathrm{HN}}+{ }^{1} D_{\mathrm{HN}}$ in the ${ }^{15} \mathrm{~N}$ dimension and by ${ }^{1} J_{\mathrm{C}^{\prime} \mathrm{C} \alpha}+$ ${ }^{1} D_{\mathrm{C}^{\prime} \mathrm{C} \alpha}$ in the ${ }^{13} \mathrm{C}^{\prime}$ dimension, yielding duplicates for each splitting and allowing an error estimate $(\sim 0.1 \mathrm{~Hz})$ from the root-mean-square value of the pairwise differences. Alignment tensors were determined by SVD fitting of the RDCs to the wild-type GB3 structure (PDB entry 2OED). ${ }^{20}$

The six alignment orientations are shown in Figure 1. The degree of linear independency of these tensors is most effectively evaluated by SVD analysis ${ }^{16}$ of the RDCs (Figure 2). The first five singular values, 97.8, 79.7, 24.9, 19.6, and $12.7 \mathrm{~Hz}$, correspond to the five independent alignment orientations spanned in our measurements. The sixth singular value $(2.5 \mathrm{~Hz})$ is five times smaller than the fifth one, indicating that perturbation to the structure and dynamics caused by the mutations is very small. A better assessment of the 


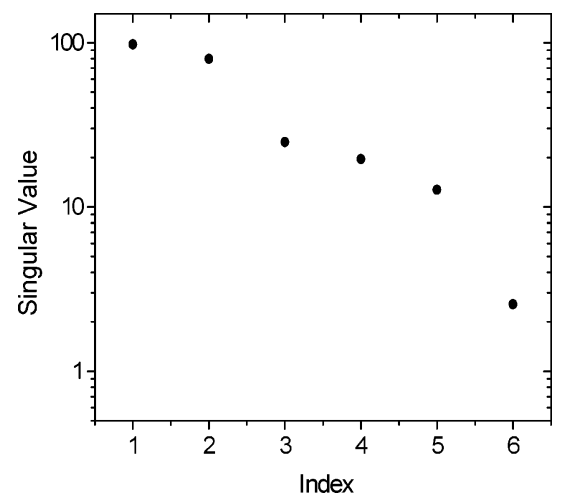

Figure 2. Singular value decomposition of ${ }^{1} D_{\mathrm{NH}} \mathrm{RDCs}$, all measured in Pf1 medium, for the six GB3 mutants. Residues 10, 11, 21, and 47, for which data in only five mutants were available, were excluded.
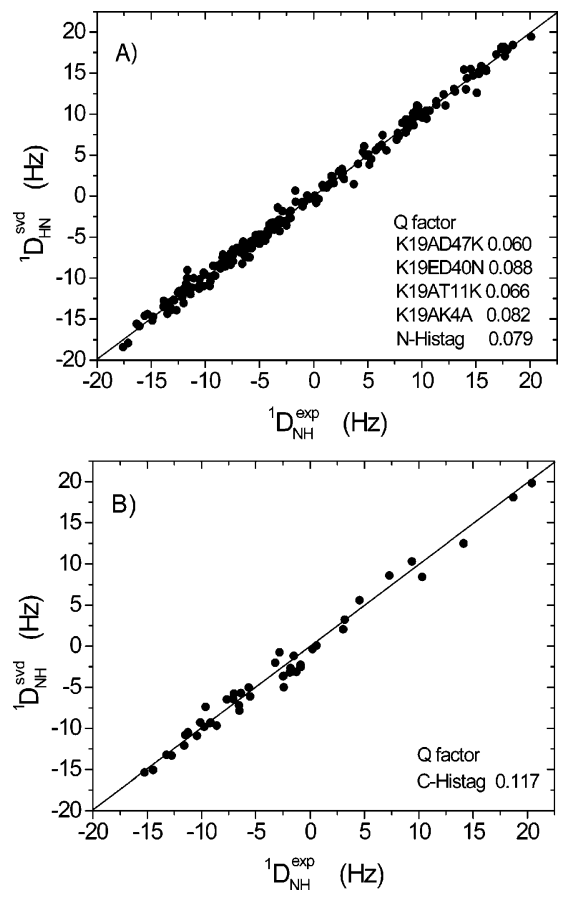

Figure 3. Correlation between experimental ${ }^{15} \mathrm{~N}-\mathrm{H}$ RDCs and couplings calculated from an SVD fit to the GB3 solution NMR structure (PDB entry 2OED) for (A) the five mutants listed in the figure and (B) for the K19EK4A C-His-tag mutants. ${ }^{1} D_{\mathrm{NH}}$ values for residues $10-12,24-26$, and $39-41$, not included in calculation of 2OED, are not included in the figures but are listed in the Supporting Information.

independence of the five alignments is obtained by SVD of the normalized alignment tensors. The corresponding singular values are $1.59,1.35,0.64,0.41$, and 0.28 for five essential mutants, while adding the sixth mutant (K19EK4A) only increases the first three singular values.

The ratio between the largest and smallest singular values is 5.7: 1 , which means that the ${ }^{1} D_{\mathrm{NH}}$ random error of $\sim 0.1 \mathrm{~Hz}$ propagates to $\sim 0.36 \mathrm{~Hz}$ for the alignment orientation with the smallest singular value, and to $\sim 0.06 \mathrm{~Hz}$ for that with the largest singular value, with others intermediate (assuming a Pf1 concentration that yields the same alignment magnitude for all five measurements).

An excellent correlation is observed between experimental RDCs and those best-fitted to the 2OED NMR structure of native
GB3, with no significant outliers for the mutated sites (Supporting Information). The corresponding $Q$ factors, ${ }^{21}$ also listed in Figure 3, confirm that any change in $\mathrm{NH}$ vector orientation between different mutants is vanishingly small. Interestingly, the fit of the K19EK4A-C-His-tag mutant RDCs is slightly worse than for the others. This alignment tensor is most orthogonal (Supporting Information) to any of the tensors used in the determination of the 2OED structure, ${ }^{20}$ suggesting that it contains new experimental information not yet present in the 2OED structure.

Our study demonstrates that mutation of charged surface groups is an effective approach to change molecular alignment without perturbing the protein backbone structure. With a set of five linearly independent alignment tensors in a single, easily manageable alignment medium, this opens the way for detailed analysis of both backbone and side-chain dynamics.

Acknowledgment. We thank Dennis A. Torchia for helpful discussions. This work was supported in part by the Intramural Research Program of the NIDDK, NIH, and by the Intramural AIDS-Targeted Antiviral Program of the Office of the Director, NIH.

Supporting Information Available: One table with ${ }^{1} D_{\mathrm{NH}}$ values in the six different GB3 mutants; one table with the normalized scalar products between the Pf1 alignment tensors of the six different mutants used in the present work, and the five tensors used in the study by Ulmer et al.; one table with products between the experimentally observed and PALES predicted tensors; one figure with residue-specific fitting errors for the six mutants. This material is available free of charge via the Internet at http://pubs.acs.org.

\section{References}

(1) Bothner-By, A. A. Encyclopedia of Nuclear Magnetic Resonance; Grant, D. M., Harris, R. K., Eds.; Wiley: Chichester, U.K., 1996; Vol. 5, pp 2932-2938

(2) Emsley, J. W. Encyclopedia of Nuclear Magnetic Resonance; Grant, D. M., Harris, R. K., Eds.; Wiley: Chichester, U.K., 1996; Vol. 4, pp 2781 2787.

(3) Tycko, R.; Blanco, F. J.; Ishii, Y. J. Am. Chem. Soc. 2000, 122, 93409341.

(4) Sass, H.-J.; Musco, G.; Stahl, S. J.; Wingfield, P. T.; Grzesiek, S. J. Biomol NMR 2000, 18, 303-309.

(5) Bouvignies, G.; Bernado, P.; Meier, S.; Cho, K.; Grzesiek, S.; Bruschweiler, R.; Blackledge, M. Proc. Natl. Acad. Sci. U.S.A. 2005, 102 13885-13890.

(6) Prestegard, J. H.; Bougault, C. M.; Kishore, A. I. Chem. Rev. 2004, 104 3519-3540.

(7) Blackledge, M. Prog. Nucl. Magn. Reson. Spectrosc. 2005, 46, 23-61.

(8) Tolman, J. R.; Ruan, K. Chem. Rev. 2006, 106, 1720-1736.

(9) Clore, G. M.; Schwieters, C. D. J. Mol. Biol. 2006, 355, 879-886.

(10) Peti, W.; Meiler, J.; Bruschweiler, R.; Griesinger, C. J. Am. Chem. Soc. 2002, $124,5822-5833$

(11) Tolman, J. R. J. Am. Chem. Soc. 2002, 124, 12020-12030.

(12) Ramirez, B. E.; Bax, A. J. Am. Chem. Soc. 1998, 120, 9106-9107.

(13) Al-Hashimi, H. M.; Valafar, H.; Terrell, M.; Zartler, E. R.; Eidsness, M K.; Prestegard, J. H. J. Magn. Reson. 2000, 143, 402-406.

(14) Hus, J. C.; Peti, W.; Griesinger, C.; Bruschweiler, R. J. Am. Chem. Soc 2003, 125, 5596-5597.

(15) Lakomek, N. A.; Carlomagno, T.; Becker, S.; Griesinger, C.; Meiler, J. J. Biomol. NMR 2006, 34, 101-115.

(16) Ruan, K.; Tolman, J. R. J. Am. Chem. Soc. 2005, 127, 15032-15033.

(17) Hansen, M. R.; Mueller, L.; Pardi, A. Nat. Struct. Biol. 1998, 5, $1065-$ 1074.

(18) Zweckstetter, M.; Hummer, G.; Bax, A. Biophys. J. 2004, 86, 34443460 .

(19) Derrick, J. P.; Wigley, D. B. J. Mol. Biol. 1994, 243, 906-918.

(20) Ulmer, T. S.; Ramirez, B. E.; Delaglio, F.; Bax, A. J. Am. Chem. Soc. 2003, 125, 9179-9191.

(21) Cornilescu, G.; Marquardt, J. L.; Ottiger, M.; Bax, A. J. Am. Chem. Soc 1998, $120,6836-6837$.

JA073937+ 